\title{
Compression Performance Study of Oil-less Helical Compressor*
}

\author{
Takayoshi FUJIWARA** Takuya HIRAYAMA** and Takashi FUKUDA** \\ ${ }^{* *}$ Compressor Engineering Department, Toshiba Carrier Corporation \\ 336 Tadewara, Fuji-shi, Shizuoka-ken, Japan \\ E-mail: takayoshi.fujiwara@toshiba.co.jp
}

\begin{abstract}
Helical compression mechanism is the newly developed one that can compress gas by using helical components. As helical shape has uneven pitch, the volume of each chamber becomes smaller along the rotation, and the gas is transferred and compressed continuously. This mechanism was designed in 1987, and the compressors with lubricant were developed and manufactured for refrigerators first in the world in 2000. And oil-less air compressors using helical mechanism were also developed and manufactured in 2003. This oil-less helical compressor doesn't contain oil at all. To achieve high performance and reliability in oil-less compressor, gas sealing, wear durability, and cooling are usually major problems, because contribution of oil to these functions can not be expected. This study focuses on the performance related to these problems, and investigates flow performance at variable motor speed, and compression process of oil-less helical compressor by measuring $P-V$ diagram.
\end{abstract}

Key words: Helical Compressor, Oil-Less, Air Compressor, Flow Performance, Compression Process, $P$ - $V$ Diagram

\section{Introduction}

There exist some mechanical structures such as reciprocating and rotary type in the positive displacement compressors. They are widely used as refrigerant compressors for refrigerators and air-conditioners. "Helical compression mechanism" mentioned in this paper is the structure that can compress gas by using helical shape, shown at Fig.1. The main parts of this mechanism consist of three parts, helical blade, roller, and cylinder, shown at left side of Fig.1. Helical blade is the part of helical shape, and the pitch is uneven along the axis. Roller is the part that has helical groove on its columnar surface. When the roller with helical blade on its surface is inserted into cylinder eccentrically, the plural compression chambers are made as shown at right side of Fig.1. While the roller revolves inside the cylinder, the gas of the compression chamber is delivered along the helical shape. Compression process of every 360 degrees is shown at Fig.2. The roller revolves, in another word "orbit", without rotation, and the helical blade stays still. As the helical shape is uneven pitch, the volume of compression chamber becomes smaller along the rotation, therefore the gas is compressed continuously.

Helical compression mechanism was designed in $1987^{(1)}$, and was manufactured first in the world in $2000^{(2)(3)}$. This mechanism has been researched for refrigerant compressor with oil since the first design, and the studies of structure, characteristics, and performance were reported in this writer's past papers ${ }^{(4)(5)(6)(7)(8)}$. As the oil can make effect on the gas sealing and lubrication, helical compressor could be confirmed to operate as refrigerant compressor.

Received 22 Dec, 2006 (No. T-04-1206) Japanese Original : Trans. Jpn. Soc. Mech. Eng., Vol.71, No.708, B (2005),

pp.2053-2058 (Received 10 Nov., 2004) [DOI: 10.1299/jee.2.115] 
Oil-less type helical compressors were developed and manufactured as air compressors in $2003^{(9)}$. The most important point of developing displacement type compressor at oil-less condition is whether the gas sealing of the chamber can operate well or not. Though the oil-use compressor can easily seal the clearance of each chamber by using oil film effect, oil-less compressor can't expect lubricant and sealing effect, and the clearance can cause the gas leakage. This paper focused on the performance of oil-less helical compressor and aimed to show its flow performance and practical compression process on oil-less condition.

\section{Nomenclature}

$P:$ pressure of chamber $e$ : eccentricity of crank $V$ : volume of chamber $V_{s}$ : displacement volume $Q$ : flow rate of discharge gas $\eta_{v}$ : volumetric efficiency

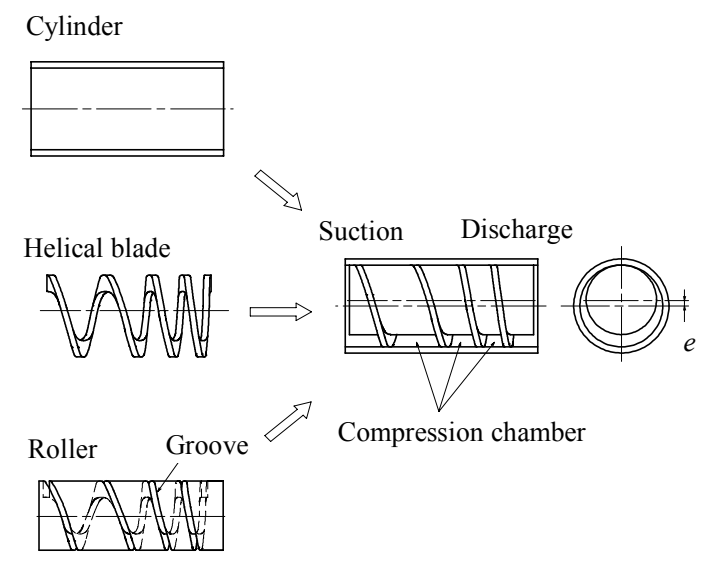

Fig.1 Main Components

$0^{\circ}$

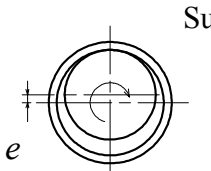

$360^{\circ}$

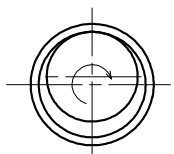

$720^{\circ}$

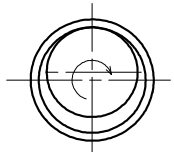

Suction

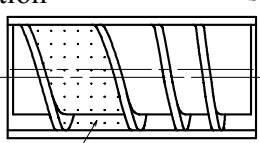

$V_{s}$

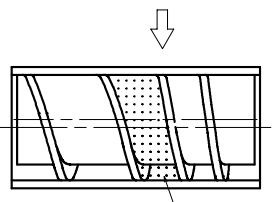

$V_{m}$

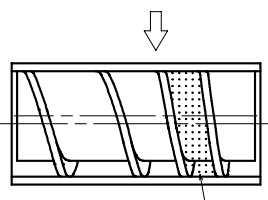

$V_{d}$

Fig.2 Compression Process 


\section{Conventional oil-less compressors}

As for the oil-less compressors of conventional displacement type, reciprocating type is the most widely used compressor, and others are rotary type compressors like rolling piston type, sliding vane type, scroll type, screw type, and so on. Reciprocating type oil-less compressor usually uses the piston ring for sealing. As the piston ring contacts with cylinder, it has high sealing ability and can make it possible to be used on high pressure condition. Whereas the advantage of reciprocating type is simple structure and low cost, the disadvantage is that the flow rate varies according to the compression ratio, and the vibration and noise is in high level because of the torque fluctuation and intermittent discharge process by the valve. On the other hand, the rotary type compressors have the characteristics that the flow rate doesn't vary according to the compression ratio so much. The attainable discharge pressure is relatively lower than that of reciprocating type, because they have some sealing clearance between the parts. Helical compressor is considered to belong to the rotary type and have some common characteristics.

\section{Required functions for Oil-less Compressors}

Whether the helical mechanism can operate and endure for long period as oil-less compressor depends on the following functions, as sealing, wear durability, and cooling.

\subsection{Sealing}

It's necessary for displacement type compressors to compose the compression chamber by preventing gas leakage. As the oil film effect for sealing can't be expected, there thought to be two measures for sealing. One is to make the moving parts contact each other. No clearance between two parts realizes good sealing performance. This measure is applied to the clearance between piston and cylinder of reciprocating compressor. The other measure is to minimize the clearance to make less the rate of leakage against the flow quantity. This measure is applied for example to the thrust clearance of rolling piston type, and at the radial clearance between orbiting and fixed scroll roller of scroll type. The helical mechanism has both way of sealing structure, contact and clearance sealing.

\subsection{Wear Durability}

At the contact sealing structure mentioned at section 3.1, wear durability of the sliding parts is important for the reliability of long life. The wear causes the expansion of clearance and then the gas leakage to deterioration of performance. The combination of these materials influences the wear durability as well as material strength itself at the sliding parts. The combination of plastic resin or carbon and the metal is usually used for sliding parts to prevent wear and damage without lubricant. The plastic resin or carbon has the role of solid lubricant. The helical blade, as the main part of helical compressor, is made of plastic resin, and moves in contact with the roller groove, and seal the gas. So the wear durability of this part is very important for reliability.

\subsection{Cooling Structure}

The work of gas compression and friction loss cause heating in compressors. In case of oil-use structure like refrigerant compressor, there is less need for cooling equipment, because the lubricant can reduce the friction loss and refrigerant gas flow has a role of cooling compressor. So the temperature of compressor can be made under tolerable level of its material without external fan. On the other hand, oil-less compressor itself has no means to cool the compressor except the gas flow. So the temperature of the parts could possibly rise over the tolerable level of its material. The overheating causes the efficiency drop and less reliability. The helical blade made of plastic resin is the most important part for cooling, 
because it is heated by compression gas, and the temperature of resin can easily rise by friction heat without oil, and the tolerable temperature is lower than that of other metal parts.

\section{Structure of Oil-less Helical compressor}

\subsection{Structure of Refrigerant Helical Compressor with Oil}

The structure of oil-use helical compressor for refrigerating equipments, has already been manufactured, is shown at Fig. $3^{(2)(3)(5)(7)}$. Both the compression parts and the motor are located inside the sealed case. The roller revolves along the crank motion, by the motor drive transmitted through the shaft. The oil in the bottom of the case is carried to the sliding parts for lubrication, gas sealing, and cooling. The oil circulation inside the sealed case is very important to secure the performance and the reliability of the compressor.

\subsection{Structure and Specification of Oil-less Compressor}

Oil-less helical compressor needs some means to ensure the performance and the reliability because there is no oil effect. The structure of oil-less helical compressor, designed to solve above mentioned problems, is shown at Fig.4, and the specification is shown at Table 1 . The compression gas is air. The displacement volume is $18 \times 10^{3} \mathrm{~mm}^{3}$. The crankshaft is driven by motor rotation. The roller revolves inside the cylinder by the crank motion. The gas is inhaled from the suction port of the cylinder, and is compressed and transferred along the helical shape, and is discharged through the exit port. The number of helical windings is 3 and the compression ratio is 2.25 , so that the air is compressed in one rotation on the basis of the study already written in the paper ${ }^{(7)}$. The motor is DC brush-less motor that can drive in speed from 20 to $50 \mathrm{~s}^{-1}$.

\subsection{Countermeasures to oil-less needs}

The sealing property is the important factor that influences the compression performance on oil-less condition. There are two sealing parts, one is the compression chamber composed of helical blade, roller, and cylinder, and the other is the thrust part of roller. The sealing of compression chambers is discussed later at section 5 . At the thrust parts of roller, the sealing part made of plastic resin is used to ensure the sealing between the chamber and the outside.

As for the wear durability of sliding parts, the material of helical blade is selected as the fluolo-carbon resin combined with filler, considering the wear durability at dry friction. Many materials were experimented by the table test, the same kind of test as oil-use condition already written in the paper ${ }^{(8)}$, because the wear durability on oil-less condition is different from that on oil-use condition. The filler was selected to achieve less wear of the material itself and less aggressive property against the other part material.

As for the cooling structure, aluminum material of good heat transfer property is used for cylinder and roller, and the cooling fan is attached to the end of the shaft, and then the holes are made through the roller to cool inside of roller by the air flow. The compression and friction heat is conducted to the cylinder and roller, and then the outside cylinder is cooled by heat radiation and external fan, and the inside roller is cooled by the internal fan. Then the temperature of helical blade made of plastic resin can be made under the tolerable level by these countermeasures. 


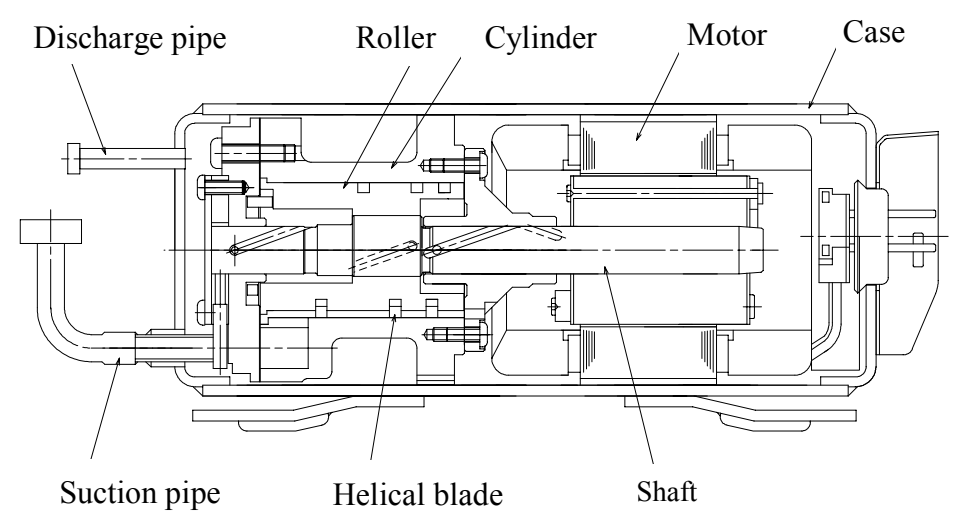

Fig.3 Refrigerant Helical Compressor Structure

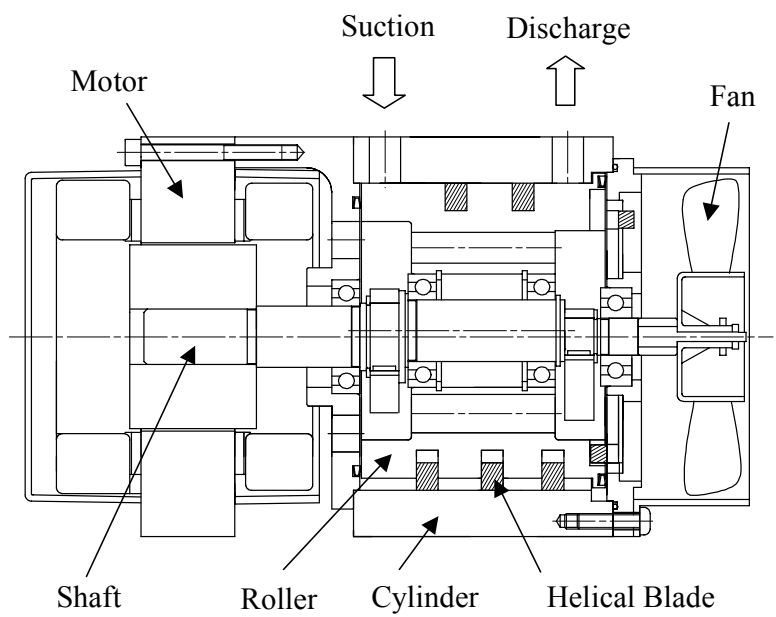

Fig.4 Structure of Oil-less Helical Compressor

Table.1 Specification of Oil-less Helical Compressor

\begin{tabular}{|l|l|}
\hline Item & Specification \\
\hline Outward Appearance & $\varphi 130 \times 231 \mathrm{~mm}$ \\
\hline Fluid & Air \\
\hline Displacement Volume & $18 \times 10^{3} \mathrm{~mm}^{3}$ \\
\hline Driving Device & DC Inverter \\
\hline Motor Speed & $20 \sim 50 \mathrm{~s}-1$ \\
\hline
\end{tabular}




\section{Sealing Structure of Compression Chamber}

The cross section of sealing structure about helical blade to divide the compression chambers is shown at Fig.5. That is very important part about sealing of oil-less helical compressor, because it influences the compression performance considerably. The helical blade divides two chambers, and the right side is the higher pressure side at Fig.5. As the helical blade is pressed to the suction side of roller groove and the inside of cylinder by the pressure difference, these contact areas are the sealing parts to divide the chambers. The sealing effect of these parts can be expected enough because of contact seal. Other sealing parts are the axial clearance between helical blade and roller, and the radial clearance between roller and cylinder. These sealing properties are considered to be influenced by the clearance. In case that the gas leakage occurs at these areas, the compression work increases by the inner leakage from the discharge side to the chamber, and the volumetric efficiency decreases by the outer leakage from the chamber to the suction side.

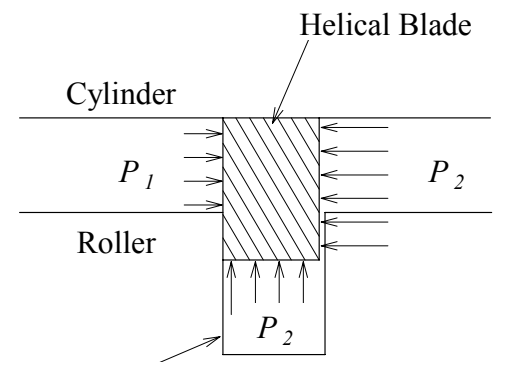

Helical Groove

Fig. 5 Cross Section of Compression chamber

\section{Flow Performance}

The flow performance of oil-less helical compressor mentioned at section 4 was measured on condition that the air was compressed by $0.1 \mathrm{MPa}$ from the atmospheric pressure. The diagram of flow rate $Q$ and volumetric efficiency $\eta_{v}$ in condition of variable rotational speed is shown at Fig.6. The volumetric efficiency $\eta_{v}$ is almost the same level as that of refrigerant compressors with oil ${ }^{(10)}$. The sealing structure mentioned at section 5 can be considered to operate well, judging from the flow performance of these test result. The flow rate $Q$ of this experiment varies in proportion to the rotational speed. The difference between the experimental flow rate and the theoretical figures shown as broken line is estimated to be equivalent to the outer leakage. The gas leakage doesn't decrease so much as the theoretical flow rate decrease from $60 \mathrm{~s}^{-1}$ to $20 \mathrm{~s}^{-1}$. Though the volumetric efficiency at rotational speed $20 \mathrm{~s}^{-1}$ is lower than that at $60 \mathrm{~s}^{-1}$, this can be considered that the leakage ratio to flow rate becomes higher at lower rotational speed.

The flow performance diagram in condition of variable discharge pressure and rotational speed is shown at Fig.7. It demonstrates that the flow rate line is almost flat in all speeds, $20,40,60 \mathrm{~s}^{-1}$, even if the discharge pressure changes. It is because that the helical compression mechanism can compress gas continuously without valve and has no residual gas, while the flow rate of reciprocating compressor varies according to the discharge pressure by re-expansion of the residual gas in the cylinder. The flow rate tends to decrease only a little according to the discharge pressure increase as shown at Fig.7. The reason is estimated that the gas leakage increases by the pressure difference. This tendency is more remarkable at lower rotational speed. 


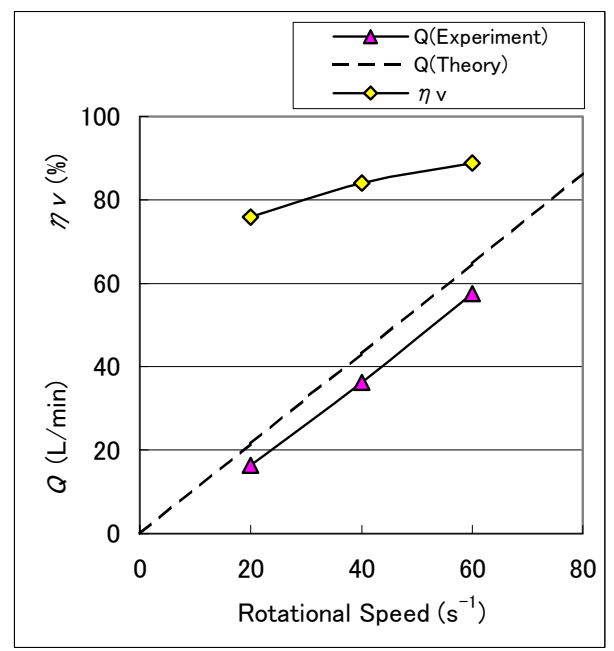

Fig. 6 Flow Performance at rotational speed

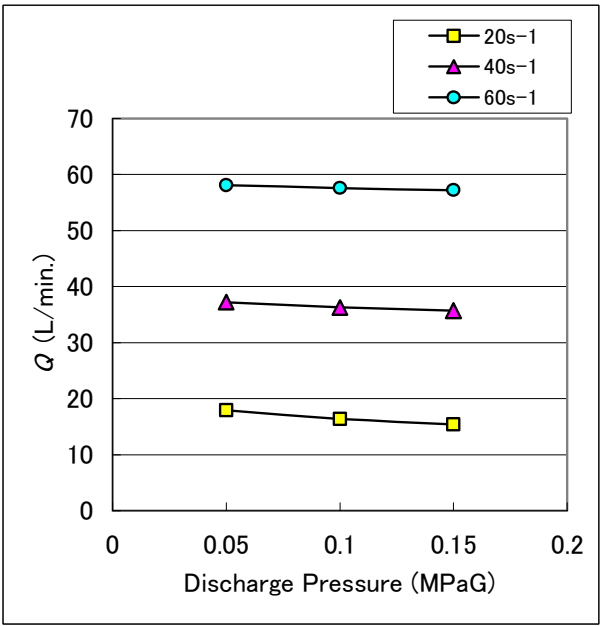

Fig. 7 Flow Performance at discharge pressure

\section{7. $P$-V Diagram}

\subsection{Method and Result of Measurement}

It's important for designing oil-less compressor to evaluate the compression process. $P-V$ diagram was led by measuring the pressure of chambers along the rotational angle. As the number of helical windings is three, the number of compression chamber is usually one, and the maximum two at rotational angle of 0 degree. So, four pressure data, those are two chambers and suction and discharge pressure, need to be measured. Four measuring holes were made along the helical shape at angle of 270 degrees intervals on the cylinder surface, and the pressure was measured with the sensors attached to these holes. Four measured data can be connected together by the analyzing recorder because the data of every 90 degrees are overlapped, and can lead to become a pressure increasing line. The compressor was operated on condition that the air was compressed by $0.1 \mathrm{MPa}$ from atmospheric pressure. So $P$ - $V$ diagram is shown as continuous line at Fig.8 and Fig.9. Fig.8 is the data of rotational speed $40 \mathrm{~s}^{-1}$, and Fig.9 is the data of $20 \mathrm{~s}^{-1}$. The theoretical line of adiabatic compression is shown as broken line to compare with experimental result.

\subsection{Study of result on condition at $40 \mathrm{~s}^{-1}$}

$P-V$ diagram of rotational speed $40 \mathrm{~s}^{-1}$ at Fig. 8 shows that the gas is compressed along the rotation and the experimental result traces near the line of adiabatic compression 
process. There is no unstable phenomenon such as expansion or excessive high pressure at the process. The experimental result shows a little higher than the theoretical line at early stage. The reason of this difference between experiment and theoretical line could be considered to be the gas leakage. As the pressure of the chamber at early stage is near the suction pressure, the gas leakage from the discharge side to the chamber is more than that from the chamber to the suction side. So the pressure of experiment tends to be higher than that of theory. And the influence of heat transfer from the cylinder to the suction gas can be also considered as that reason.

As for the discharge process, Fig. 8 shows a little over compression phenomenon. As the compression ratio by the helical design is 2.25 , the gas is over compressed in the chamber on condition that the pressure ratio is 2 . And the over expansion phenomenon of suction process can't be seen at Fig.8. This characteristic is almost the same as the helical compressor for refrigerant with oil. The reason can be considered that the pass area is kept enough for suction gas flow during the whole suction process.

\subsection{Study of result on condition at $20 \mathrm{~s}^{-1}$}

$P-V$ diagram of rotational speed $20 \mathrm{~s}^{-1}$ at Fig.9 shows that the difference between experiment and theoretical line at compression process is larger than that of $40 \mathrm{~s}^{-1}$. As this tendency is shown at whole compression process, the quantity of gas leakage could be considered more than that of $40 \mathrm{~s}^{-1}$. According to the result of performance measurement at section 6 , the gas leakage rate per time is estimated almost the same at every rotational speed. So, as the gas leakage per one rotation at speed $20 \mathrm{~s}^{-1}$ becomes more than that at

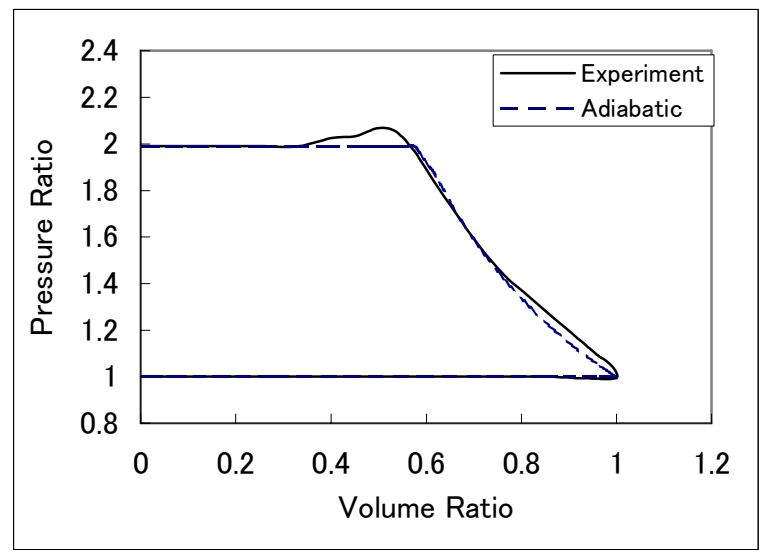

Fig. $8 \quad P$ - $V$ Diagram $\left(\right.$ at $\left.40 \mathrm{~s}^{-1}\right)$

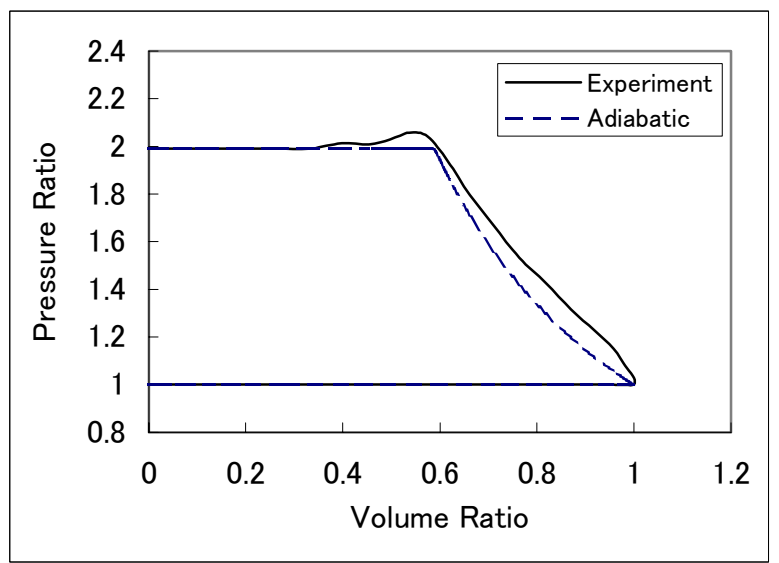

Fig. $9 \quad P$-V Diagram $\left(\right.$ at $\left.20 \mathrm{~s}^{-1}\right)$ 
$40 \mathrm{~s}^{-1}$, it is estimated that influences the experimental result of $P-V$ diagram.

The ratio of loss against the whole compression work could be considered to be small enough for achieving high efficiency, judging from the study of $P-V$ diagram. So it is confirmed that the sealing structure of helical mechanism mentioned at section 5 can operate well as oil-less compressor.

\section{Conclusion}

Oil-less air compressor using helical mechanism was newly developed. This paper shows the key points of this development, like flow performance and compression process of oil-less helical compressor. It could be testified that the flow rate of oil-less helical compressor is proportional to the rotational speed and doesn't depend on the pressure condition so much, by the test that the compressor was driven on compression ratio 2 and rotational speed from $20 \mathrm{~s}^{-1}$ to $60 \mathrm{~s}^{-1}$. The volumetric efficiency is almost the same level as that of refrigerant compressors with oil. As the result of $P-V$ diagram, the pressure of chamber increases along the rotation, and the experimental compression line traces near the adiabatic compression process. Neither phenomenon of over compression at discharge process nor over expansion at suction process is remarkable. So it could be confirmed that the ratio of these losses against the whole compression work is small enough for achieving high efficiency. By the result of flow performance and $P-V$ diagram, it could be verified that the sealing structure of helical mechanism operates well as oil-less compressor.

\section{References}

(1)Fujiwara, T. \& Iida, T. 1987, 'Fluid Compressor', Patent: H7-107391

(2)Aikawa, E. \& Fujiwara, T. et al, 2001, 'Development and product commercialization of Helical compressor', The Japan Society of Mechanical engineers, Vol.104, No.990(2001.5), pp265

(3)Nikkei Mechanical, No. 555(2000.12), pp65

(4)Iida, T. \& Fujiwara, T. et al, 1993, 'Development of New Compression Mechanism', Japan Society of Refrigerating and Air Conditioning Engineers Vol.10, No.3, pp.475-482

(5)Okuda, M. \& Fujiwara, T. et al, 2001, 'Development of New Compression Mechanism', Japan Society of Refrigerating and Air Conditioning Engineers, pp.9-12

(6)Fujiwara, T. \& Aikawa, E. et al, 1998, 'Analysis of New type Compressor', Proceedings of the 1998 International Compressor Engineering Conference at Purdue, pp.81-86

(7)Fujiwara, T. \& Okuda, M. et al, 2005, 'Helical Structure of Helical Compression Mechanism', Transactions of The Japan Society of Mechanical engineers, Vol. 71, pp.100-105

(8)Fujiwara, T. \& Oyama, S. 2004, 'Reliability Study of Helical Compression Mechanism', Transactions of The Japan Society of Mechanical engineers, Vol.70, pp.197-202

(9)Fukuda, T. \& Okuda, M. et al, 2003, 'Oil-less Helical Compressor', Toshiba Review, Vol.58, No.10, pp.70-73

(10)Refrigerating and Air conditioning Handbook, Basic, Japan Refrigerating Association, pp. 12 